POS PROCEEDINGS

\title{
Polarized synchrotron emission
}

\author{
Carlo Burigana* \\ INAF-IASF Bologna \\ Via Gobetti 101, I-40129, Bologna, Italy \\ E-mail: burigana@iasfbo.inaf.it
}

\section{Laura La Porta; Wolfgang Reich, Patricia Reich}

Max-Planck-Institut für Radioastronomie

Auf dem Hügel, 69, D-53121 Bonn, Germany

E-mail: laporta@mpifr-bonn.mpg.de, wreich@mpifr-bonn.mpg.de,

preich@mpifr-bonn.mpg.de

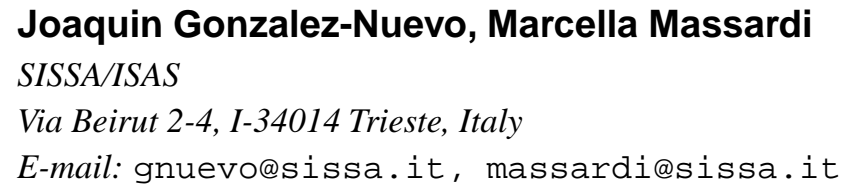

Gianfranco De Zotti

INAF - Osservatorio Astronomico di Padova

Vicolo dell'Osservatorio 5, I-35122 Padova, Italy

E-mail: dezotti@pd.astro.it

Galactic synchrotron emission represents the most relevant foreground contamination in cosmic microwave background (CMB) anisotropy observations at angular scales $\theta \gtrsim 1^{\circ}$ and frequencies $v \lesssim 70 \mathrm{GHz}$. The accurate understanding of its polarization properties is crucial to extract the cosmological information contained in the $\mathrm{CMB}$ polarization anisotropy. Radio surveys at $v \sim 1 \mathrm{GHz}$ offer the unique opportunity to study Galactic synchrotron emission where it represents the dominant component, possibly except for regions close to the Galactic plane where free-free emission is also important. We review the observational status of Galactic radio surveys at scales $\theta \gtrsim 0.5^{\circ}$. Leiden surveys, thanks to their frequency coverage from $0.408 \mathrm{GHz}$ to $1.411 \mathrm{GHz}$, still remain of fundamental importance for the comprehension of depolarization phenomena. Recent surveys at $1.42 \mathrm{GHz}$ (in both total intensity and polarization) with a better sensitivity and sky sampling now cover both celestial hemispheres and allow to accurately map the correlation properties of the diffuse synchrotron emission. We present an analysis of these surveys in terms of angular power spectrum. A comparison of a simple frequency extrapolation of these results with the recent WMAP results shows that we are close to map the bulk of the diffuse synchrotron polarization fluctuations and to understand the corresponding implications for CMB experiments.

$C M B$ and Physics of the Early Universe

20-22 April 2006

Ischia, Italy

\section{*Speaker.}

${ }^{\dagger}$ Member of the International Max Planck Research Shool (IMPRS) for Radio and Infrared Astronomy at the 


\section{Introduction}

The Galactic polarized diffuse synchrotron radiation is expected to play the major role at frequencies below $70 \mathrm{GHz}$ on intermediate and large angular scales $\left(\theta \gtrsim 30^{\prime}\right)$, at least at medium and high Galactic latitudes where satellites have the clearest view of the CMB anisotropies. At about $1 \mathrm{GHz}$ the synchrotron emission is the most important radiative mechanism out of the Galactic plane, while at low latitudes it is comparable with the bremsstrahlung; however the free-free emission is unpolarized, whereas the synchrotron radiation could reach a theoretical intrinsic degree of polarization of about $75 \%$ (see [17]). Consequently, radio frequencies are the natural range for studying it (see [42]), though it might be affected by Faraday rotation and depolarization.

It is standard practice to characterize the CMB anisotropies in terms of angular power spectrum ([34, 22, 56]) (APS) as a function of the multipole, $\ell$ (inversely proportional to the angular scale, $\ell \simeq 180 / \theta\left(^{\circ}\right)$ ), in total intensity ( $T$ mode), polarization ( $P I, E$, and $B$ modes), and crosscorrelation modes (in particular, we will consider the $T E$ mode). Although the APS is certainly not exhaustive to fully characterize the complexity of the intrinsically non-Gaussian Galactic emission, it is of increasing usage also to characterize the Galactic foreground correlation properties in both data analysis and theoretical studies (see e.g. [1, 11, 12]). Using the anafast facility of the HEALP ix ${ }^{1}$ package by [18], we computed the APS of the above modes for the various considered surveys, sky areas, and frequencies.

\section{Analysis of the Leiden polarization surveys}

Nowadays the only available radio data suitable for multifrequency studies on large scales are the so called Leiden surveys ([5]), globally covering a sky fraction of about $\sim 50 \%$, mainly in the northern Galactic hemisphere. These linear polarization surveys are the result of different observational campaigns carried out in the sixties with the Dwingeloo 25-m radio telescope at 408, 465, 610,820 and $1411 \mathrm{MHz}$ with angular resolutions respectively of $\theta_{H P B W}=2.3^{\circ}, 2.0^{\circ}, 1.5^{\circ}, 1.0^{\circ}, 0.6^{\circ}$. The complete data sets as well as the observations reduction and calibration methods have been presented by [5]. Specific interpolation methods to project these surveys into HEALPix maps with pixel size $\simeq 0.92^{\circ}$ (see Fig. 1) have been recently implemented ([27]), catching with the main problem of these surveys, i.e. their poor average sampling across the sky and their limited sensitivity ( $\sim 0.34,0.33,0.16,0.11$ and $0.06 \mathrm{~K}$ from the lower to the higher frequency, respectively). Such methods have been successfully tested against simulations.

Three sky areas with sampling significantly better than the average (by a factor $\simeq 4 ;[1,6,27]$ ) permit to reach multipoles $\ell \simeq 100$ : patch $1\left[\left(110^{\circ} \leq l \leq 160^{\circ}, 0^{\circ} \leq b \leq 20^{\circ}\right)\right]$; patch $2\left[\left(5^{\circ} \leq\right.\right.$ $\left.l \leq 80^{\circ}, b \geq 50^{\circ}\right)$ together with $\left(0^{\circ} \leq l \leq 5^{\circ}, b \geq 60^{\circ}\right)$ and $\left.\left(335^{\circ} \leq l \leq 360^{\circ}, b \geq 60^{\circ}\right)\right]$; patch $3\left[\left(10^{\circ} \leq l \leq 80^{\circ}, b \geq 70^{\circ}\right)\right]$. These patches are associated with the brightest structures of the polarized radio sky, i.e. the Fan Region (patch 1) and the North Polar Spur (NPS) (patch 2 and 3). The NPS has been extensively studied (see $[43,15]$ ). The theories that better meet the observations interprete the NPS as the front shock of an evolved Supernova Remnant (SNR), whose distance should be $\sim 100 \pm 20 \mathrm{pc}$ (inferred from starlight polarization, see [3]). In contrast, the present

Universities of Bonn and Cologne.

${ }^{1}$ http://healpix.jpl.nasa.gov/ 

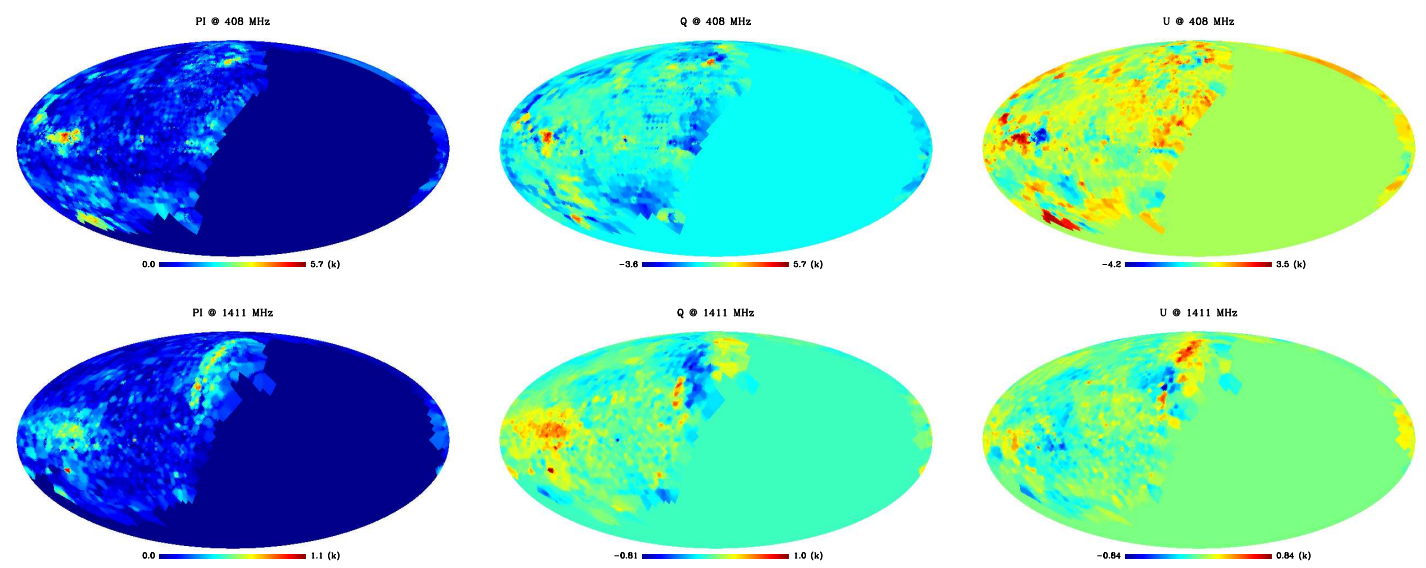

Figure 1: $P I, Q$, and $U$ maps at $408 \mathrm{MHz}$ and $1411 \mathrm{MHz}$ obtained from the Leiden surveys data tables. Adapted from [27].
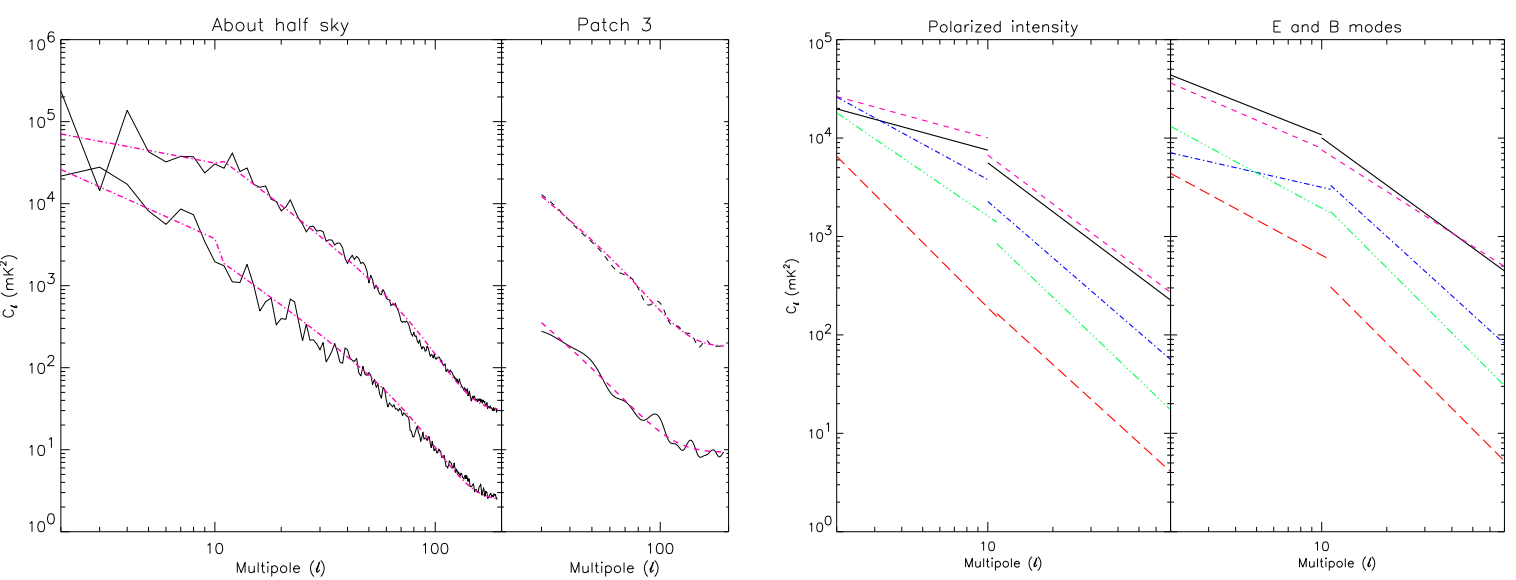

Figure 2: Left panels: polarization APSs at $610 \mathrm{MHz}$ (solid lines) for the survey full coverage (left panel) and patch 3 (right panel) together with the corresponding best fit curves (dot-dash lines). The lower curves in each panel are $C_{\ell}^{P I}$, while the other ones represent $C_{\ell}^{E ; B}$ multiplied by 10 . Right panels: synchrotron component of the APS derived for the survey full coverage for the polarized intensity (left panel) and for $C_{\ell}^{E ; B}$ (right panel) at the various frequencies: 408 (solid line), 465 (dashes), 610 (dot-dash), 820 (three dot-dash), and $1411 \mathrm{MHz}$ (long dashes). Adapted from [27].

knowledge of the Fan Region is much poorer. A rough estimate of its distance can be derived from purely geometrical considerations: it has such an extent on the sky that it must be located within $(1-2) \times 10^{2} \mathrm{pc}$ from the Sun not to have unrealistically large dimension (see also [54]). Another common and remarkable characteristic of the selected areas is that the polarization vectors appear mostly aligned at the two higher frequencies of the Leiden surveys. Another region (patch 4) located at $\left(70^{\circ} \leq l \leq 120^{\circ},-45^{\circ} \leq b \leq-15^{\circ}\right)$ with a sky sampling in the average (its APS is then statistically relevant only for $\ell \lesssim 50$ ) but characterized by a rather low signal has been also considered by [27]. 


\subsection{Angular power spectra}

In general, the $E$ and $B$ modes turned out to be extremely similar ${ }^{2}$, so that, instead of studying each of them singularly, it can be more advantageous to consider their average $C_{\ell}^{E ; B}=\left(C_{\ell}^{E}+C_{\ell}^{B}\right) / 2$ ([27]). In some cases we could even consider a unique APS defined as $\left(C_{\ell}^{P I}+C_{\ell}^{E}+C_{\ell}^{E}\right) / 3$. The maps essentially represent the Galactic polarized synchrotron emission, smoothed with the beam of the radiotelescope and contaminated by the noise. As a consequence, their APSs can be fit as the sum of two components, $C_{\ell}=C_{\ell}^{\text {synch }} W_{\ell}+C_{\ell}^{\mathrm{N}}$. We exploit the power law approximation $C_{\ell}^{\text {synch }} \simeq \kappa \cdot \ell^{\alpha}$ and assume a symmetric, Gaussian beam, i.e. a window function $W_{\ell}=\mathrm{e}^{-\left(\sigma_{b} \ell\right)^{2}}$, where $\sigma_{b}=\theta_{H P B W}(\mathrm{rad}) / \sqrt{8 \ln 2}$. Under the hypothesis of uncorrelated Gaussian random noise (white noise), $C_{\ell}^{\mathrm{N}}=C_{\ell}^{\mathrm{WN}} \sim$ const. The fit has been performed on the multipole range $[30,200]$ for the patches and $[2,200]$ for the full-coverage maps ([27]). In fact, the flattening occurring at higher multipoles helps the recovering of the noise constant, in spite of the fact that no reliable astrophysical information can be derived for $\ell>100$ from the APSs even in the three better sampled regions ([6]). For the patches, a single set of the parameters $\kappa, \alpha$ allows to describe the APS in the entire range of multipoles. In the case of the survey full coverage two sets of $\kappa, \alpha$ are needed, each of them appropriate to a certain multipole range. In fact, at all frequencies a change in the APS slope occurs in correspondance of $\ell \sim 10$ for both $C_{\ell}^{P I}$ and $\left(C_{\ell}^{E}+C_{\ell}^{B}\right) / 2$. As an example, Fig. 2 displays the APS and the corresponding best fit curve at $610 \mathrm{MHz}$ for the survey full coverage and one patch. It shows also the recovered synchrotron term, $C_{\ell}^{\text {synch }}$, for the survey. Table 1 lists the values of $\kappa, \alpha$ obtained for the patches. In the multipole range $30 \lesssim \ell \lesssim 100$ a general trend appears: from the lowest to the highest frequency the slope steepens from $\sim-(1-1.5)$ to $\sim-(2-3)$, with a weak dependence on the considered sky region ([27]).

\begin{tabular}{|c|c|c|c|c|c|c|c|c|c|c|}
\hline & \multicolumn{2}{|c|}{$408 \mathrm{MHz}$} & \multicolumn{2}{c|}{$465 \mathrm{MHz}$} & \multicolumn{2}{c|}{$610 \mathrm{MHz}$} & \multicolumn{2}{c|}{$820 \mathrm{MHz}$} & \multicolumn{2}{c|}{$1411 \mathrm{MHz}$} \\
& $\kappa\left(\mathrm{mK}^{2}\right)$ & $-\alpha$ & $\kappa\left(\mathrm{mK}^{2}\right)$ & $-\alpha$ & $\kappa\left(\mathrm{mK}^{2}\right)$ & $-\alpha$ & $\kappa\left(\mathrm{mK}^{2}\right)$ & $-\alpha$ & $\kappa\left(\mathrm{mK}^{2}\right)$ & $-\alpha$ \\
\hline 1 & $9.80 \cdot 10^{4}$ & 0.99 & $6.00 \cdot 10^{5}$ & 1.50 & $2.21 \cdot 10^{6}$ & 2.05 & $3.08 \cdot 10^{6}$ & 2.59 & $9.80 \cdot 10^{5}$ & 2.90 \\
& $1.00 \cdot 10^{5}$ & 1.10 & $6.00 \cdot 10^{5}$ & 1.67 & $9.44 \cdot 10^{6}$ & 2.69 & $2.02 \cdot 10^{6}$ & 2.64 & $9.80 \cdot 10^{5}$ & 2.90 \\
\hline 2 & $1.26 \cdot 10^{6}$ & 1.81 & $2.50 \cdot 10^{6}$ & 1.95 & $2.00 \cdot 10^{7}$ & 2.66 & $2.58 \cdot 10^{6}$ & 2.56 & $1.85 \cdot 10^{6}$ & 3.13 \\
& $1.26 \cdot 10^{6}$ & 1.10 & $1.00 \cdot 10^{5}$ & 1.32 & $3.10 \cdot 10^{5}$ & 1.82 & $2.00 \cdot 10^{6}$ & 2.68 & $1.85 \cdot 10^{6}$ & 3.13 \\
\hline 3 & $8.00 \cdot 10^{4}$ & 1.12 & $1.81 \cdot 10^{7}$ & 2.61 & $1.65 \cdot 10^{6}$ & 2.09 & $1.52 \cdot 10^{6}$ & 2.56 & $1.70 \cdot 10^{5}$ & 2.45 \\
& $1.20 \cdot 10^{5}$ & 1.61 & $8.00 \cdot 10^{5}$ & 1.32 & $9.00 \cdot 10^{5}$ & 2.28 & $3.53 \cdot 10^{6}$ & 2.93 & $2.70 \cdot 10^{5}$ & 2.10 \\
\hline 4 & $2.00 \cdot 10^{4}$ & 0.91 & $2.60 \cdot 10^{4}$ & 1.08 & $3.10 \cdot 10^{6}$ & 2.69 & $3.36 \cdot 10^{6}$ & 3.07 & $6.10 \cdot 10^{5}$ & 2.97 \\
& $2.00 \cdot 10^{4}$ & 0.91 & $1.10 \cdot 10^{5}$ & 1.65 & $2.60 \cdot 10^{5}$ & 2.24 & $7.00 \cdot 10^{4}$ & 2.28 & $1.00 \cdot 10^{6}$ & 3.33 \\
\hline
\end{tabular}

Table 1: Least-square best fit parameters $\kappa$ and $\alpha$ obtained in the APS analysis of the patches. Relative errors on the best fit parameters are $\sim 10 \%$. The considered multipole range is [30-200]. For each patch, the first (second) line refers to $C_{\ell}^{P I}\left(C_{\ell}^{E ; B}\right)$. Adapted from [27].

\footnotetext{
${ }^{2}$ On the contrary, the $E$ and $B$ modes of the primordial CMB anisotropies differ largely as they are induced by different mechanisms (e.g. [44], [25].
} 


\section{Multifrequency APS analysis of Faraday depolarization effects}

The theoretical intrinsic behaviour of the synchrotron total intensity emission predicts a power law dependence of the APS amplitude on frequency: $T^{\text {synch }} \propto v^{-\beta} \Rightarrow C_{\ell}^{\text {synch }}(v) \propto v^{-2 \beta}$, where $\beta=\delta+2$. The values of $\delta$ deduced from radio observations change with the considered sky position and range between $\sim 0.5$ and $\sim 1$ (see $[39,36]$ ). In principle, one would expect the synchrotron emission to be highly polarized, up to a maximum percentage of $\sim 75 \%$ ([17]). In the ideal case of constant degree of polarization the above formulae would apply also to the polarized component of the synchrotron emission, once the brightness temperature has been properly rescaled. However, depolarization effects are relevant at radio frequencies and can mask a possible correlation between total and polarized intensity. The current knowledge of $\beta$ for the Galactic diffuse polarized emission is very poor, but already indicates that due to depolarization phenomena the observed $\beta$ can be much lower than $\sim 2.7$ (e.g. for the NCP [52] quote $\beta \sim 1.87$ ). From the values of $C_{\ell=\tilde{\ell}}^{\text {synch }}(v)$ at various $\ell$, the APS amplitude is found to decrease with frequency, but this frequency dependence is weaker than that observed in total intensity, as expected in the presence of a frequency dependent depolarization ([27]).

An electromagnetic wave travelling through a magnetized plasma will undergo a change in the polarization status. Its polarization vector will be rotated by an angle $\Delta \phi[\mathrm{rad}]=R M\left[\mathrm{rad} / \mathrm{m}^{2}\right]$. $\lambda^{2}\left[\mathrm{~m}^{2}\right]$, where the rotation measure, $R M$, is the line of sight integral $R M\left[\mathrm{rad} / \mathrm{m}^{2}\right]=0.81 \int n_{e}\left[\mathrm{~cm}^{-3}\right]$. $B_{\|}[\mu \mathrm{G}] d l[\mathrm{pc}]$, being $n_{e}$ the electron density and $B_{\|}$the component of the magnetic field along the line of sight. In the slab model ([9]), differential rotation of the polarization angle decreases the polarized intensity (Faraday depolarization) and the observed and intrinsic polarization intensities are related by $T^{p, o b s}(\lambda)=T^{p, i n t r}(\lambda) \cdot|\sin \Delta \phi / \Delta \phi|$. Being $\left(T^{p}\right)^{2} \propto C_{\ell}(v)$, the above formula reads: $C_{\ell}(v)=C_{\ell}^{\text {intr }}(v) \cdot(\sin \Delta \phi / \Delta \phi)^{2}$, where $C_{\ell}^{\text {intr }}(v)=\kappa \cdot \ell^{\alpha} \cdot v^{-2 \beta}$. At a given $\ell$, considering two frequencies we have: $C_{\ell}\left(v_{1}\right) / C_{\ell}\left(v_{2}\right)=\left(v_{1} / v_{2}\right)^{-2 \beta} \cdot\left(\Delta \phi_{2} / \Delta \phi_{1}\right)^{2} \cdot\left(\sin \Delta \phi_{1} / \sin \Delta \phi_{2}\right)^{2}$. Given $\beta$, the expression on the right side of this equation can be computed as a function of $R M$ and the observed value of $C_{\ell}\left(v_{1}\right) / C_{\ell}\left(v_{2}\right)$ can provide informations about $R M$.

If the polarization angles vary within the beam area, then observations of the same sky region with different angular resolution will give an apparent change of the polarized intensity with frequency because of beamwidth depolarization; on the contrary, bandwidth depolarization is negligible for these surveys (see $[16,45])$. Suppose $\theta_{H P B W, 1}$ and $\theta_{H P B W, 2}\left(<\theta_{H P B W, 1}\right)$ are the angular resolutions respectively at $v_{1}$ and $v_{2}$. The effect of beamwidth depolarization on $C_{\ell}\left(v_{1}\right) / C_{\ell}\left(v_{2}\right)$ can be removed smoothing the map at the frequency $v_{2}$ to the (lower) angular resolution of the map at the frequency $v_{1}$ or, equivalently in practice, multiplying the observed APSs ratio, $C_{\ell}\left(v_{1}\right) / C_{\ell}\left(v_{2}\right)$ by $\mathrm{e}^{\left(\Delta \sigma_{b} \ell\right)^{2}}$, where $\Delta \sigma_{b}=\sigma_{1}{ }^{2}-\sigma_{2}^{2}\left(\sigma_{i}=\theta_{H P B W, i}(\operatorname{rad}) / \sqrt{8 \ln 2} ; i=1,2\right)$.

Therefore, from $C_{\tilde{\ell}}^{\text {synch }}(820 \mathrm{MHz}) / C_{\tilde{\ell}}^{\text {synch }}(1411 \mathrm{MHz})$ (case in which the oscillations of this theoretical ratio do not introduce the dramatic degeneration appearing at lower frequencies), the following intervals of $R M$ are found to be compatible with the data: $\simeq 9-17,53-60,75-87,123$ $130 \mathrm{rad} / \mathrm{m}^{2}$ together with two narrow intervals at $\simeq 70$ and $140 \mathrm{rad} / \mathrm{m}^{2}$ ([27]). A typical $R M$ value of $\simeq 8 \mathrm{rad} / \mathrm{m}^{2}$ has been reported by [47], however this is likely a lower limit to the real values. The possible values of the $R M s$ can be also constrained by the observed polarization degree, defined as $\Pi=T^{p} / T$. The mean polarization degree $\bar{\Pi}$ in the portion of the sky covered by the Leiden survey at $1411 \mathrm{MHz}$ can be estimated exploiting the total intensity northern sky map at $1.4 \mathrm{GHz}([41,38])$ : 

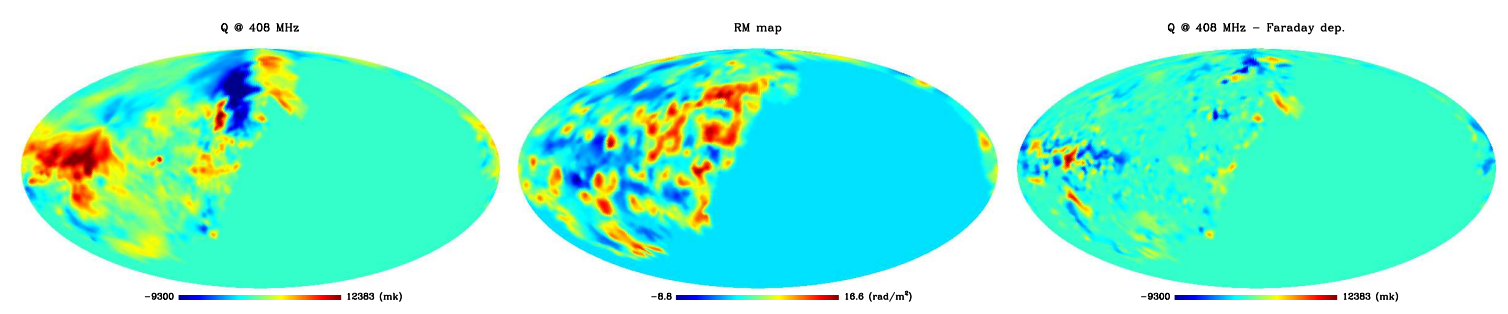

Figure 3: Maps of the $Q$ Stokes parameter at $408 \mathrm{MHz}$ before (left panel) and after (right panels) the transformation for Faraday depolarization through the $R M$ map (middle panel) used in the simulation. Similar results are found for the $U$ Stokes parameter. All maps have been smoothed to 2.3 ${ }^{\circ}$. Adapted from [27].

$\bar{\Pi}^{\text {obs }} \sim 17,27,28,25,12 \%$ for the full coverage and for the patch 1,2,3, and 4, respectively. Given the relation between the intrinsic and observed brightness polarized temperature in the slab model approximation, we have: $\Pi^{\text {obs }}=\Pi^{\text {intr }} \cdot|\sin \Delta \phi / \Delta \phi|$. The maximum possible value of $\Pi^{\text {obs }}$ (derived assuming $\Pi^{\text {intr }}=0.75$ ) can be computed as function of the $R M$ and compared with the mean polarization degree actually observed in the considered cases ${ }^{3}$. The observed polarization degree sets the upper limit $R M \lesssim 50 \mathrm{rad} / \mathrm{m}^{2}$, consistent with the lowest of the $R M$ intervals previously identified ([27]).

As already shown, the synchrotron emission APS steepens with increasing frequency. One possible explanation of this behaviour again relies on depolarization arguments: electron density and/or magnetic field fluctuations (in strength and/or direction) might redistribute the synchrotron emission power from larger to smaller angular scales, creating fake structures, the effect being less important at increasing frequency. Sticking to the ISM slab model, the Faraday depolarization effects on the APS at $408 \mathrm{MHz}$ and $1420 \mathrm{MHz}$ can be simulated as follows. The $1420 \mathrm{MHz}$ polarization map ([54]) is assumed to represent the intrinsic Galactic synchrotron emission and is scaled down to $408 \mathrm{MHz}$ with a spectral index of -2.75 . The intrinsic polarization angle, as mapped by the polarization angle map at $1420 \mathrm{MHz}$, should be the same at the two frequencies. An RM map constructed exploiting the catalog produced by T.A.T. Spoelstra (private communication), consisting of $\sim 1000 R M$ data, has been adopted to transform the intrinsic $\phi$ and $P I$ for Faraday rotation and differential Faraday depolarization: $\phi_{\text {out put }} \rightarrow \phi_{\text {input }}+\Delta \phi$ and $P I_{\text {out put }} \rightarrow P I_{\text {input }} \cdot|\sin (\Delta \phi) / \Delta \phi|$. At $1420 \mathrm{MHz}$ the input and output maps are very similar. At $408 \mathrm{MHz}$ the large scale structure appears significantly depressed in the depolarized map, whereas the small scale features become much more relevant. The results of this toy-simulation are shown in Fig. 3 for the Stokes parameter $Q$. The comparison between the APSs of the input and output maps quantifies this behaviour ([27]): at $1420 \mathrm{MHz}$ the APSs are almost identical while at $408 \mathrm{MHz}$ the depolarized map APSs significantly flatten, in agreement with the derived APS flattening with decreasing frequency reported in Table 1.

\footnotetext{
${ }^{3}$ Note that the observed mean polarization degree might be due to the combination of Faraday depolarization and beam depolarization. In case we were able to correct for beam depolarization effects this analysis will lead to smaller values of RMs. Therefore the results of our analysis are rather conservative and provide upper limits to the RMs compatible with observations in the considered patches.
} 


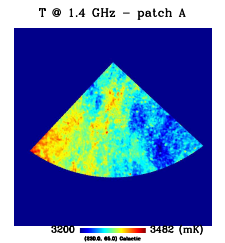

PI 14.4 GH - patcl $A$

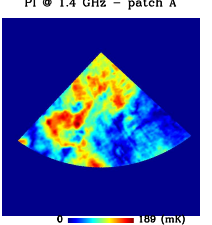

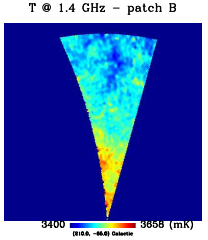

PI $\oplus 1 / 4 \mathrm{GH} 2-$ patcl B

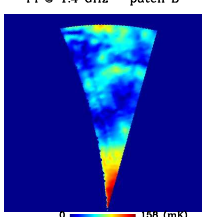

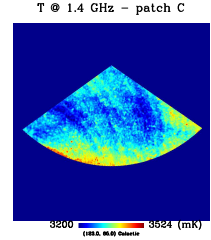

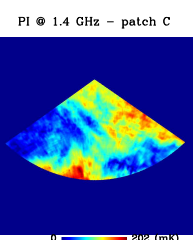

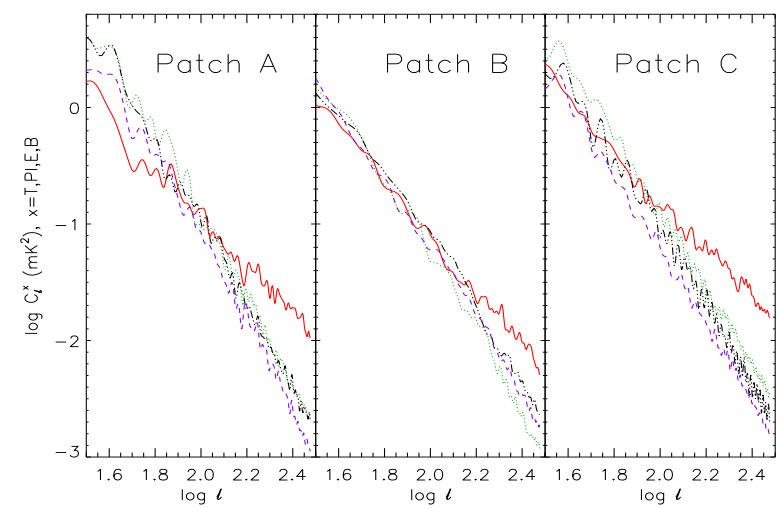

Figure 4: Left small panels: gnomonic projection of the considered cold patches in total intensity (top panels) and polarization (bottom panels). Right panels: APSs of the selected cold regions (solid lines (red lines) $\rightarrow C_{\ell}^{T}$; dashes (blue lines) $\rightarrow C_{\ell}^{P I}$; dots (green lines) $\rightarrow C_{\ell}^{E}$; three dot-dashes (black lines) $\rightarrow C_{\ell}^{B}$ ). Adapted from [28].

\section{Analysis of the DRAO 1.4 GHz polarization survey}

A new linear polarization survey of the northern celestial hemisphere at $1.42 \mathrm{GHz}$ with an angular resolution $\theta_{H P B W} \simeq 36^{\prime}$ has been recently completed using the DRAO $26 \mathrm{~m}$ telescope ([54]). The survey has a spacing, $\theta_{s}$, of $15^{\prime}$ in right ascension and from $0.25^{\circ}$ to $2.5^{\circ}$ in declination, which requires interpolation to construct equidistant cylindrical (ECP) maps with $\theta_{\text {pixel }}=15^{\prime}$ ([53]). The final maps have an angular resolution of about $36^{\prime}$ and an rms-noise of about $12 \mathrm{mK}$, which is unique so far in terms of sensitivity and coverage. These polarization data have been used by [28] in combination with the Stockert total intensity map at $1.42 \mathrm{GHz}$ ([41, 38]), having the same angular resolution, similar sensitivity, and $\theta_{s}=15^{\prime}$.

\subsection{Selected areas and results}

Being interested in the diffuse Galactic synchrotron emission, [8] subtracted discrete sources (DSs) from the total intensity map (only very few sources are visible in polarization out of the plane) performing a 2-dimensional Gaussian fitting (NOD2-software library, [19]). We are confident that at $|b| \gtrsim 45^{\circ}$ DSs with peak flux densities exceeding $\sim 1$ Jy have been removed that way (see [8] for a map of the subtracted DSs).

We then derived the APSs in both temperature and polarization for the whole DRAO survey coverage and the three following sky areas (see Fig. 4) characterized by a relatively weak polarized intensity: patch A $-180^{\circ} \leq l \leq 276^{\circ}, b \geq 45^{\circ}$; patch B $-193^{\circ} \leq l \leq 228^{\circ}, b \leq-45^{\circ}$; patch C $65^{\circ} \leq l \leq 180^{\circ}, b \geq 45^{\circ}$. In all patches the polarization APSs are rather similar ([28]). This fact might indicate that depolarization due to differential Faraday rotation should not be relevant in such regions of the sky with respect to the investigated angular scales ([27]). In fact, rotation measure (RM) maps ([21, 13]) obtained interpolating RM data of extragalactic sources show very low values in correspondence of such areas. However, the degree of polarization is on average a few percent, well below the maximum theoretical value of $\sim 75 \%$. The reason for the low fractional polarization is not clear. One possibility is that depolarization other than differential is present (e.g. [45]). 


\begin{tabular}{|c|c|c|c|c|}
\hline \multicolumn{2}{|c|}{ Coverage } & \multicolumn{3}{|c|}{ Best Fit parameters } \\
\hline \multicolumn{2}{|c|}{} & $\log _{10} \kappa\left(\mathrm{mK}^{2}\right)$ & $\alpha$ & $c^{s r c}\left(\mathrm{mK}^{2}\right)$ \\
\hline $\mathrm{C}$ & $C_{\ell}^{T}$ & $4.339_{+0.284}^{-0.297}$ & $-2.64_{-0.01}^{+0.03}$ & $0.047_{+0.030}^{-0.013}$ \\
& $C_{\ell}^{P I}$ & $4.748_{+0.350}^{-0.650}$ & $-2.94_{-0.36}^{+0.20}$ & $0.004_{+0.004}^{-0.004}$ \\
& $C_{\ell}^{E ; B}$ & $4.954_{+0.444}^{-0.301}$ & $-2.94_{-0.26}^{+0.20}$ & $0.005_{+0.009}^{-0.005}$ \\
\hline
\end{tabular}

Table 2: Least-square best fit parameters describing the total intensity and polarization APSs for the patch $\mathrm{C}$ in the range $30 \leq \ell \leq 300$. The errors are given so that the upper (resp. lower) values correspond to the best fit parameters set with the flattest (resp. steepest) slope. Adapted from [28].

The APSs (namely $C_{\ell}^{T}, C_{\ell}^{P I}$ and $C_{\ell}^{E ; B}$ ) can be modelled as $C_{\ell}=\left(C_{\ell}^{\text {synch }}+c^{s s c}\right) \cdot W_{\ell}+c^{W N}$ ([28]). The power law approximation $C_{\ell}^{\text {synch }} \simeq \kappa \cdot \ell^{\alpha}$ has been exploited. The contribution of non-subtracted DSs has been modelled with a constant term according to the formalism of Poisson fluctuations from extragalactic point sources ([49]). From extragalactic source counts at $1.4 \mathrm{GHz}$ (see e.g. [37]) we estimate $c^{s r c} \sim 0.09 \mathrm{mK}^{2}$ for the total intensity case. We assumed again a symmetric Gaussian beam window function. A first guess estimate of the noise term $c^{W N}$ is provided by $C_{\ell}^{W N} \sim 4 \pi \cdot \sigma_{p i x} / N_{p i x}$, where $N_{p i x}$ is the number of pixels in the HEALPix map and $\sigma_{p i x}=\sigma_{p i x, \mathrm{HEALPix}} \sim N^{-1 / 2} \cdot \sigma_{p i x, \mathrm{ECP}}$ with $N$ number of the ECP pixels corresponding to each HEALP ix pixel at a given latitude.

The resulting best fit parameters for one patch are listed in Table 2 . The parameter range for the Galactic synchrotron emission APS is $\alpha \sim[-2.71,-2.60]$ for the slope and $\log _{10} \kappa \sim[4.067,4.339]$ $\left(\kappa\right.$ in $\mathrm{mK}^{2}$ ) for the amplitude. The DS contribution is in the range $c_{s r c} \sim[0.023,0.047] \mathrm{mK}^{2}$, which is consistent, within the errors, with the above source count estimate ${ }^{4}$. For polarization, the derived slopes are generally steeper, varying in the interval $\alpha \sim[-3.02,-2.62]$ for $C_{\ell}^{P I}$ and $\alpha \sim$ $[-3.05,-2.55]$ for $C_{\ell}^{E ; B}$ ([28]). The DS contribution is on average much lower than in temperature, being clearly compatible with zero ${ }^{5}$. The possibility to apply a source subtraction exploiting dedicated point source templates ([31]) produced from existing catalogs is under investigation.

\section{Local analysis of the all-sky surveys}

In the recent years a complete coverage of the radio sky at $1420 \mathrm{MHz}$, both in total intensity and in polarization intensity, has been achieved. It derives from the combination of the Stockert total intensity survey $([41,38])$, of two DRAO polarization survey previously discussed, and of the Villa Elisa total intensity ([40]) and polarization ([48]) survey which covers the southern celestial hemisphere with the same resolution, similar sensitivity, and with a uniform sampling $\left(\theta_{s}=15^{\prime}\right)$.

\footnotetext{
${ }^{4}$ The bulk of discrepancy of a factor $\sim 2$ between the value of $c^{s r c}$ recovered by our fit and that predicted from best fit number counts can be in fact produced by the survey sky sampling $\left(\theta_{S}\right)$ of $15^{\prime}$.

${ }^{5}$ The best fit results may suggest a polarization degree (obtained considering also the contribution of the subtracted DSs, $\sim 0.05-0.2 \mathrm{mK}^{2}$, to the temperature APS) considerably higher than $\sim 2 \%$, the value found for NVSS extragalactic (mainly steep spectrum) sources $([32,50])$. It may imply a presence (or a combination) of spurious instrumental polarization at very small scales, of a significant contribution from highly polarized Galactic sources ([30]) non-subtracted in the maps, or of a flattening of the diffuse synchrotron polarized emission APS at $\ell \gtrsim 200-250$ with respect to the behaviour at $\ell \lesssim 200-250$, as that found in higher resolution analyses on smaller sky areas $([1,10])$.
} 

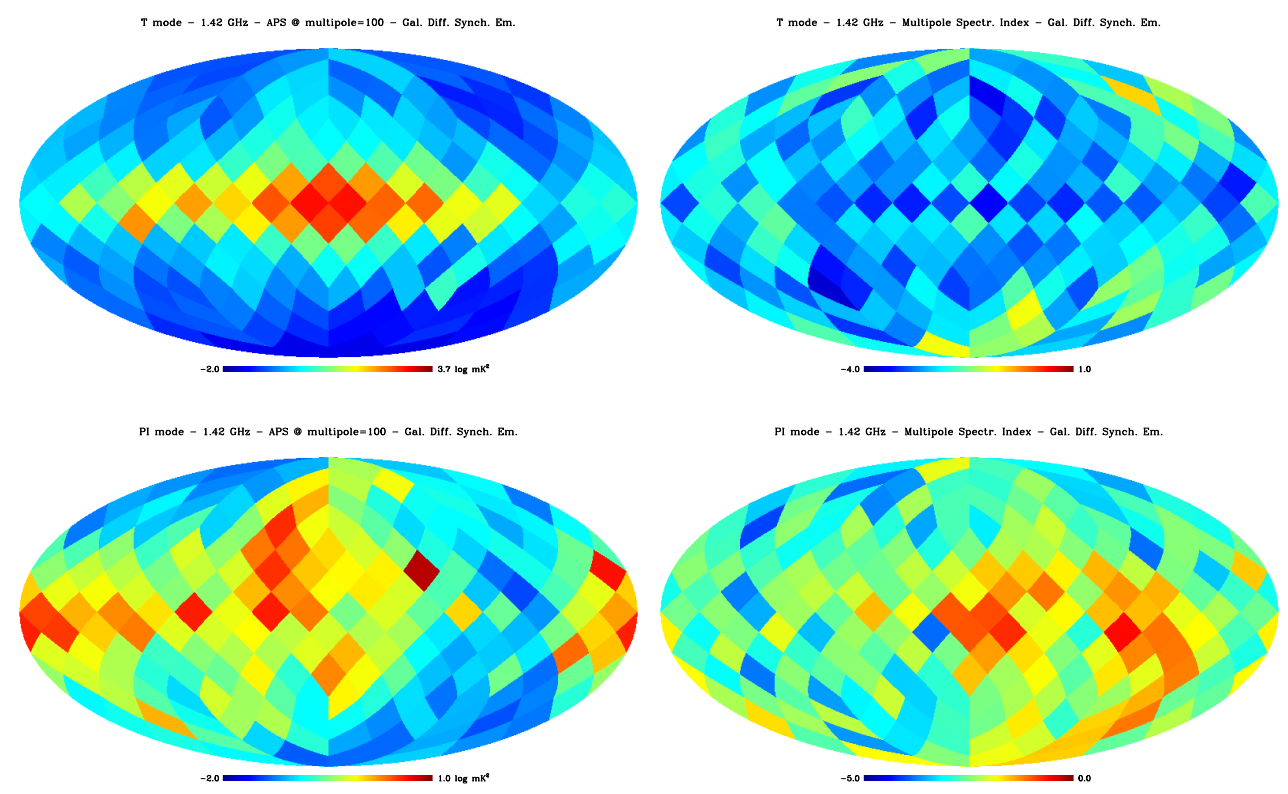

Figure 5: Results of the local APS analysis for the total and polarization intensity maps. The results for the $E$ and $B$ modes are very similar to those found for $P I$.

A first APS analysis of the preliminary version of these all-sky surveys for representative Galactic cuts has been presented in [8]. Great efforts have been recently dedicated to the intercalibration of the various sky areas and further improvements are forseen. The present analysis is based on an updated version of the all-sky polarization survey. As evident, diffuse synchrotron correlation properties depend on the considered sky areas. We present here preliminary results of the first APS local analysis on the whole sky $([29,26])$, previously anticipated in [7]. By using HEALPix tessellation, we divide the sky in patches of a size of $\simeq 14.7^{\circ}$ which allows to accurately compute the APSs ${ }^{6}$ at $\ell \gtrsim 60$ (while angular resolution limits the analysis to $\ell \lesssim 300$ ). We then fit the APSs with the same parametrization of Sect. 4.1, by previously removing bright discrete sources in total intensity as in Sect. 4.1. The result is shown in Fig. 5. Clearly, both the APS amplitude (reported here at a reference multipole $\ell=100$ ) and the spectral index exibit very different patterns in total intensity and polarization, much more related to the Galactic latitude in the former case. In total intensity we find a certain APS steepening for increasing power while this kind of correlation does not appear in polarization, or, in other words, the region at relatively higher power and flatter APS in the $C_{\ell=100^{-}} \alpha$ plane is more populated in polarization than in total intensity.

\section{Comparison with CMB polarization anisotropy}

The accurate measure of the $E$ mode is of particular relevance for breaking the existing degeneracy in cosmological parameter estimation when only temperature anisotropy data are available (e.g. $[4,14])$. The detection of the primordial $B$ mode is of fundamental importance for testing

\footnotetext{
${ }^{6}$ We derived the $T$ and PI APSs both passing through the computation of the angular correlation function and directly with anafast to check the reliability of the found results.
} 

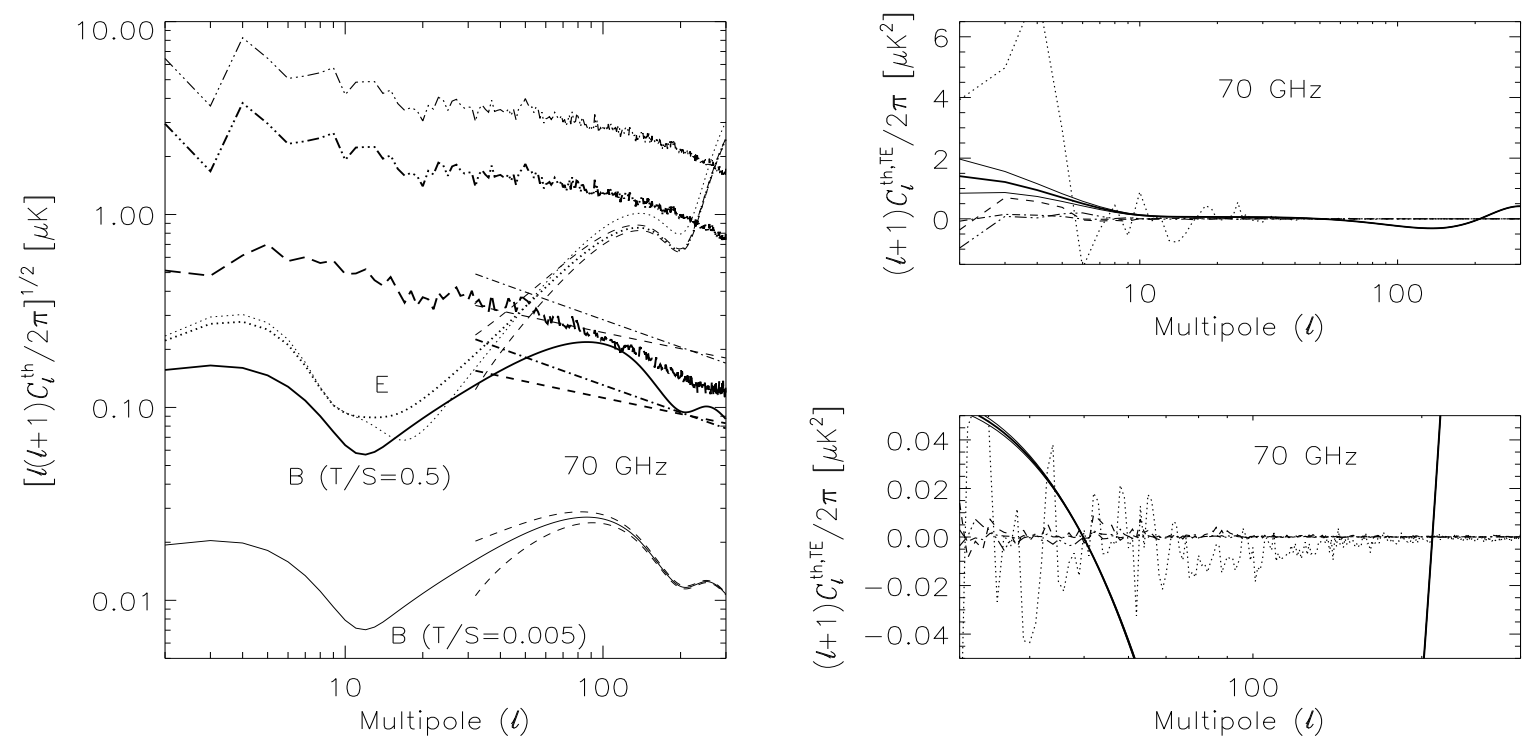

Figure 6: Comparison between the $E$ and $B$ modes (right panel) and the $T E$ mode (left panels) of the CMB anisotropy at $70 \mathrm{GHz}$ and of the Galactic polarized synchrotron foreground. Dotted and solid lines: CMB $E$ and $B$ mode for two different tensor to scalar ratios (thin line $T / S=0.005$, thick line $T / S=0.5$ ). Dashed lines: uncertainty associated to the cosmic and sampling variance for a sky coverage of $\sim 3 \%$ and a binning of $10 \%$ in $\ell$. Three dots-dashes: average of the $E$ and $B$ modes extrapolated from the whole DRAO survey for the spectral indices $\beta=-2.8$ and -3 , respectively. The anafast results have been divided by the window function to correct for beam smoothing. Thick long dashed line: the same as above but with a mask at $|b| \leq 20$ for $\beta=-3$. Dashed (dot-dashed) power law: best fit result corresponding to Patch B (resp. C), rescaled in frequency as above. The CMB $T E$ mode (thick solid line) is for $T / S=0.005$; the corresponding cosmic variance (without binning in $\ell$ ) identified by the thin solid lines assumes all-sky coverage. Dots: extrapolated DRAO TE mode for a spectral index $\beta=-3$. Dashes (dot-dashes): as above but masking the region at $|b| \leq 5^{\circ}$ and adopting $\beta=-2.8(\beta=-3)$. Three dots-dashes: as above but excluding the region at $|b| \leq 20^{\circ}(\beta=-2.8)$. Top and bottom panels are identical but with a different choice of the multipole and power range, for a better view of the results. The results are in terms of thermodynamic temperature, as usual in CMB studies. Adapted from [28].

the existence of a stochastic cosmological background of gravitational waves (e.g. [23]). The frequency range $60-80 \mathrm{GHz}$ seems the less contaminated by Galactic synchrotron and dust foregrounds in both temperature ([2]) and polarization ([33]) at angular scales $\gtrsim 1^{\circ}$.

We have extrapolated the APS for the $E$ and $B$ modes derived from the DRAO survey analysis to $70 \mathrm{GHz}$ and compared it with the APS of the CMB polarization anisotropy for a $\Lambda \mathrm{CDM}$ model including scalar and tensor perturbations compatible with the recent WMAP 3 years results ${ }^{7}$ ([46]). The results of this comparison ([28]) are displayed in Fig. 6 for two choices of the temperature spectral index $(\beta=-2.8,-3)$. The APS extrapolated from the entire DRAO survey is also shown. It exceeds the CMB $E$ mode even at the peak at $\ell \sim 100$ for $\beta=-2.8$. For $\beta=-3$ the two APSs are almost comparable. Fig. 6 shows also the APS extrapolated from the DRAO survey excluding the region $|b| \leq 20^{\circ}$ and adopting $\beta=-3$. Such a sky mask reduces the Galactic APS

\footnotetext{
${ }^{7}$ http://lambda.gsfc.nasa.gov/product/map/current/
} 
below the CMB $E$ mode for $\ell \gtrsim 50$. In this case the CMB $B$ mode peak at $\ell \sim 100$ is comparable to (or exceeds) the synchrotron signal for tensor to scalar ratios $T / S \gtrsim 0.5$. For the three patches and $T / S \gtrsim 0.5$ the cosmological $B$ mode peak at $\ell \sim 100$ is comparable to (or exceeds) the Galactic synchrotron signal extrapolated with $\beta \simeq-2.8$, while it is larger by a factor $\gtrsim 2$ (in terms of $\sqrt{C_{\ell}}$ ) for $\beta \simeq-3$. Furthermore, a separation of the Galactic synchrotron polarized signal from the CMB signal with an accuracy of $\sim 5-10 \%$ (in terms of $\sqrt{C_{\ell}}$ ) would allow to detect the CMB $B$ mode peak at $\ell \sim 100$ even for $T / S \sim 0.005$ if $\beta \simeq-3$. Similar results for the detection of the B mode peak at $\ell \sim 100$ have been obtained by [10] based on $1.4 \mathrm{GHz}$ Effelsberg polarization observations of a small region with exceptionally low Galactic foreground contamination, though at $\ell \sim$ few $\times 100$ the CMB $B$ mode is expected to be dominated by the $B$ mode generated by lensing ([57]). We note also that for a sky coverage comparable with those of the considered patches $(\sim 3 \%)$ the cosmic and sampling variance does not significantly limit the accuracy of the CMB mode recovery at $\ell \gtrsim$ some $\times 10$.

The CMB $T E$ correlation sets constrains on the reionization history from the power at low multipoles and on the nature of primordial fluctuations from the series of peaks and antipeaks. In Fig. 6 we compare the $T E$ mode APS of the Galactic emission extrapolated at $70 \mathrm{GHz}$ with the CMB one. The APS of the whole DRAO survey indicates a significant Galactic contamination at $\ell \lesssim$ few $\times 10$ even for $\beta \simeq-3$. The use of a proper Galactic mask (e.g. excluding regions with $|b| \leq 5^{\circ}$ for $\beta \sim-3$ or $|b| \leq 20^{\circ}$ for $\beta \sim-2.8$ ) largely reduces the Galactic foreground contamination even at low multipoles. For the three cold patches the Galactic TE mode is fully negligible compared to the $\mathrm{CMB}$ one independently of the adopted $\beta$. The $T E$ mode antipeak at $\ell \sim 150$ turns out to be very weakly affected by Galactic synchrotron contamination in all cases. These considerations ([28]) further support the reliability of the $T E$ mode measure performed by WMAP and of the derived conclusions on the epoch of reionization and of a dominance of adiabatic perturbations and existence of superhorizon temperature fluctuations at decoupling $([24,33])$ confirming the inflationary paradigm ([35]).

The comparison of WMAP $3 y$ r results on polarized foregrounds with those derived from radio surveys is of particular interest in the case of all-sky coverage. For a better comparison, we applied to the all-sky $1.4 \mathrm{GHz}$ polarization map the same polarization mask considered by the WMAP team, which excludes $\simeq 24 \%$ of sky (mainly the Galactic plane and the NPS). In this comparison, we exploit a first order correction for Faraday depolarization by increasing the $1.4 \mathrm{GHz}$ polarized APS of a factor $1 / 0.85$ corresponding to a typical value of $R M \sim 15 \mathrm{rad} / \mathrm{m}^{2}$, as inferred from Sect. 3, before applying a simple power law frequency extrapolation with constant $\beta$ to WMAP and PLANCK ${ }^{8}$ frequencies. The result is shown in Fig. 7. It can be easily compared with Fig. 17 of [33]. Clearly, radio data are more sensitive to synchrotron emission. This opportunity reflects into the more accurate and regular APS shape, particularly at $\ell \gtrsim 10$ where the intercalibration of the various sky areas is less critical. At $v<40 \mathrm{GHz}$ there is a good agreement between the average APSs extrapolated from radio surveys and those derived with WMAP, which includes the overall contribution of polarized foregrounds. In spite of the difficulty to fully understand the complexity of ISM structure and depolarization effects because of the role of various physical processes (tur-

\footnotetext{
${ }^{8}$ http://www.rssd.esa.int/planck
} 


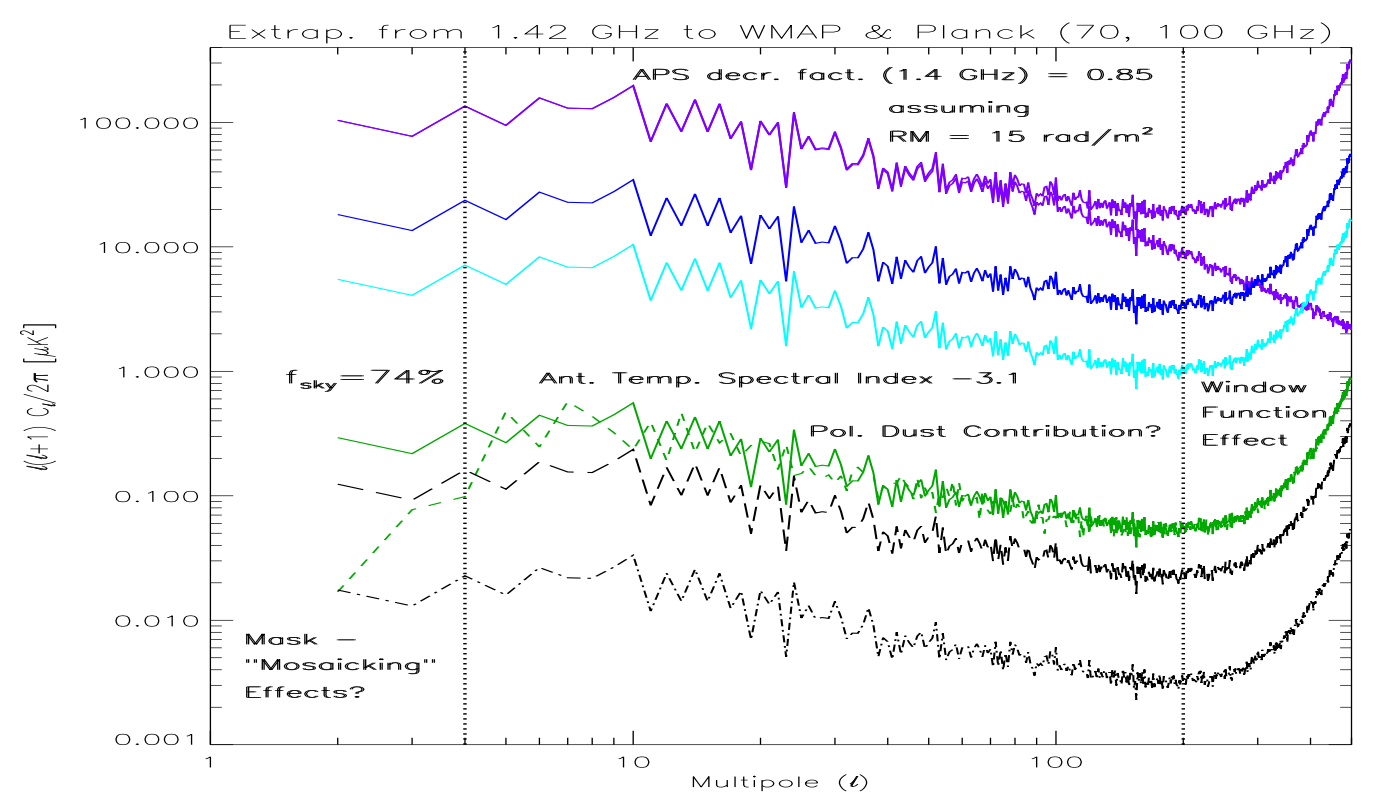

Figure 7: Galactic synchrotron APSs ( $E$ mode, except for (green) short dashes that refer to $B$ mode at $60.3 \mathrm{GHz}$, for comparison) of the Galactic synchrotron emission extrapolated to $v / \mathrm{GHz}=22.8,30.3,36.9$, $60.3,70,100$ (from top to bottom) from the $1.4 \mathrm{GHz}$ all-sky survey applying the WMAP polarization mask. For a better comparison with Fig. 17 of [33], the APSs have been divided by the window function to account for beam smoothing and partially correct the power at $\ell \sim 100-200$, but the power increasing at $\ell \gtrsim 200$ is clearly non-physical (at $22.8 \mathrm{GHz}$ the smoothed APS is also shown).

bulences in both the Galactic magnetic field and the ISM electron density depolarizing the diffuse synchrotron emission (see [20]), foreground magneto-ionic structures (Faraday screens) modulating the background synchrotron diffuse emission (see [55, 51]), probably more relevant at smaller scales), this agreement supports the idea that the bulk of the correlation properties of the microwave synchrotron polarized emission at $\ell \lesssim 300$ can be mapped on the basis of radio data resorting to relatively simple Faraday depolarization arguments and frequency rescaling. On the contrary, in the $\mathrm{V}$ band the WMAP foreground APS is about a factor of two above that extrapolated from radio surveys. If not explained by an unlikely significant frequency flattening of the synchrotron polarized emission at $v \gtrsim 40 \mathrm{GHz}$ with respect to the behaviour at $v \lesssim 40 \mathrm{GHz}$, this discrepancy can be only explained in terms of additional polarized contributions in the $\mathrm{V}$ band (with a global power similar to that of the diffuse synchrotron emission), to be probably ascribed to polarized dust emission.

Acknowledgements - We warmly thank R. Beck, P. Leahy, T.A.T. Spoelstra, L. Toffolatti, M. Tucci, and R. Wielebinski for useful discussions. Some of the results in this paper have been derived using HEALPix ([18]). The use of the CMBFAST code (version 4.5.1) is acknowledged. L.L.P. was supported for this research through a stipend from the International Max Planck Research School (IMPRS) for Radio and Infrared Astronomy at the universities of Bonn and Cologne.

\section{References}

[1] Baccigalupi, C., Burigana, C., Perrotta, F., et al., 2001, A\&A, 372, 8 
[2] Bennett, C. L., Hill, R. S., Hinshaw, G., et al., 2003, ApJS, 148, 97

[3] Bingham, R. G., 1967, MNRAS, 137, 157

[4] Bond, J. R., Davis, R. L., Steinhardt, P. J., 1995 ApL\&C, 32, 53

[5] Brouw, W. N., Spoelstra, T. A. T., 1976, A\&AS, 26, 129

[6] Burigana, C., La Porta, L., 2002, in Astrophysical Polarized Background, AIP Conf. Proc. 609, 54, astro-ph/0202439

[7] Burigana, C., La Porta, L., Reich, P., Reich, W., 2005, talk at the Workshop "Polarisation 2005 - Sky polarisation at far-infrared to radio wavelengths: The Galactic Screen before the Cosmic Microwave Background", University Paris-XI campus, September 12-15, 2005

[8] Burigana, C., La Porta, L., Reich, P., Reich, W., 2006, AN, 327, No. 5/6, 491

[9] Burn, B. J. 1966, MNRAS, 133, 67

[10] Carretti, E., Poppi, S., Reich, W., et al., 2006, MNRAS, 367, 132

[11] Chepurnov, A. V., 1999, Astron. Astrophys. Trans., 17, 281

[12] Cho, J., Lazarian, A., 2002, ApJ, 575, L63

[13] Dineen, P., Coles, P., 2005, MNRAS, 362, 403

[14] Efstathiou, G., Bond, J. R., 1999, MNRAS, 304, 75

[15] Egger, R. J., Aschenbach, B., 1995, A\&A, 294, L25

[16] Gardner, F. F., Whiteoak, J. B., 1966, ARA\&A, 4, 245

[17] Ginzburg, V. L., Syrovatski, S. I., 1965, ARA\&A, 3, 297

[18] Górski, K. M., Hivon, E., Banday, A. J., et al., 2005, ApJ, 622, 759

[19] Haslam, C. G. T., 1974, A\&AS,15,333

[20] Haverkorn, M., Katgert, P., de Bruyn, A. G., 2004, A\&A, 427, 549

[21] Johnston-Hollitt, M., Hollitt, C. P., Ekers, R. D., 2004, in The Magnetized Interstellar Medium, ed. B. Uyaniker, W. Reich, R. Wielebinski (Copernicus GmbH), 13, http://www.mpifr-bonn.mpg.de/div/konti/antalya/contrib.html

[22] Kamionkowski, M., Kosowsky, A., Stebbins, A., 1997, Phys.Rev. D, 55, 7368

[23] Knox, L., Turner, M. S., 1994, PhRvL, 73, 3347

[24] Kogut, A., 2003, NewAR, 47, 977

[25] Kosowsky, A., 1999, NewAR, 43, 157

[26] La Porta, L., 2006, PhD Thesis, Bonn University, Germany, in prep.

[27] La Porta, L., Burigana, C., 2006, A\&A, in press, astro-ph/0601371

[28] La Porta, L., Burigana, C., Reich, W., Reich, P., 2006, A\&AL, in press, astro-ph/0606xxx

[29] La Porta, L., Burigana, C., Reich, W., Reich, P., 2006, in prep.

[30] Manchester, R. N., Han, J. L., Qiao, G. J., 1998, MNRAS, 295, 280

[31] Massardi, M., Gonzalez-Nuevo, J., De Zotti, G., 2006, this conference 
[32] Mesa, D., Baccigalupi, C., De Zotti, G., et al., 2002, A\&A, 396, 463

[33] Page, L., Hinshaw, G., Komatsu, E., et al., 2006, ApJ, submitted, astro-ph/0603450

[34] Peebles, P. J. E., 1993, Principles of Physical Cosmology, Princeton University Press

[35] Peiris, H. V., Komatsu, E., Verde, L., et al., 2003, ApJS, 148, 213

[36] Platania, P., Bensadoun, M., Bersanelli, M., et al., 1998, ApJ, 505, 2, 473

[37] Prandoni, I., Gregorini, L., Parma, P., et al., 2001, A\&A, 365, 392

[38] Reich, P., Reich, W., 1986, A\&AS, 63, 205

[39] Reich, P., Reich, W., 1988, A\&AS, 74, 7

[40] Reich, P., Testori, J. C., Reich, W., 2001, A\&A, 376, 861

[41] Reich, W., 1982, A\&AS, 48, 219

[42] Reich, W., 2006, in Review Book “Cosmic Polarization”, ed. R. Fabbri, Publisher: Research Signpost, astro-ph/0603465

[43] Salter, C. J., 1983, BASI, 11, 1

[44] Seljak, U., Zaldarriaga, M., 1997, Phys. Rev. Lett., 78, 2054

[45] Sokoloff, D. D., Bykov, A. A., Shukurov, A., et al., 1998, MNRAS, 299, 1985

[46] Spergel, D. N., Bean, R., Dore, O., et al., 2006, ApJ, submitted, astro-ph/0603449

[47] Spoelstra, T. A. T., 1984, A\&AS, 135, 238

[48] Testori, J. C., Reich, P., \& Reich, W. 2003, in The Magnetized Interstellar Medium, ed. B. Uyaniker, W. Reich, and R. Wielebinski (Katlenburg-Lindau: Copernicus GmbH), 57, http://www.mpifr-bonn.mpg.de/div/konti/antalya/contrib.html

[49] Toffolatti, L., Argüeso Gómez, F., De Zotti, G., et al., 1998, MNRAS, 297, 117

[50] Tucci, M., Martínez-González, E., Toffolatti, L., et al. 2004, MNRAS, 349, 1267

[51] Uyaniker, B., 2003, in The Magnetized Interstellar Medium, ed. B. Uyaniker, W. Reich, R. Wielebinski (Katlenburg-Lindau: Copernicus GmbH), 71, http://www.mpifr-bonn.mpg.de/div/konti/antalya/contrib.html

[52] Vinyajkin, E. N., Razin, V. A., 2002, in Astrophysical Polarized Background, AIP Conf. Proc. 609, 26

[53] Wolleben, M., 2005, PhD Thesis, Bonn University, Germany

[54] Wolleben, M., Landecker, T. L., Reich, W., Wielebinski, R., 2006, A\&A, 448, 411

[55] Wolleben, M., Reich, W., 2004, A\&A, 427, 537

[56] Zaldarriaga, M., 2001, Phys. Rev. D, 64, 15

[57] Zaldarriaga, M., Seljak, U., 1998, Phys. Rev. D, 58, 3003 